Supporting Information:

\section{Analysis of chain branch of polyolefins by a new proton NMR approach}

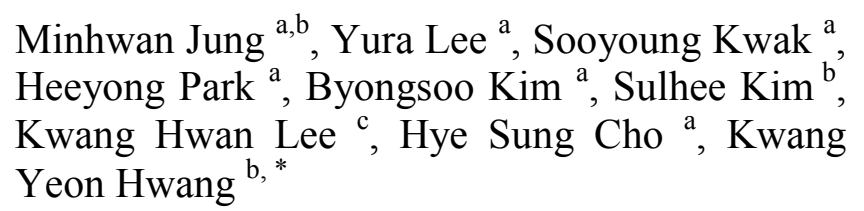

${ }^{a}$ Corporate R\&D, LG Chem Research Park, 104-1, Moonji-dong, Yuseung-gu, Daejeon, 305-380, Republic of Korea.

${ }^{\mathrm{b}}$ Department of Biosystems \& Biotechnology, Division of Biotechnology, Korea University, Seoul, 136-713, Republic of Korea.

${ }^{\mathrm{c}}$ Bruker Korea, 4F. KT Net building, Pangyoro 338, Bundang-Gu, Seongnam-si, Gyeonggi-do, 463-847, Republic of Korea.

*Corresponding author: chahong $@$ korea.ac.kr

1. Below are the parameters employed for NMR analysis:

1) Proton NMR (zg30) : 16 scans, delay of $3 \mathrm{~s}, 90$ degree pulse length of $13.5 \mu \mathrm{s}$ at $21 \mathrm{~W}(-13.2 \mathrm{~dB})$.

2) Homo-decoupling NMR (zghd.2 or zghd.3) : 8 scans, delay of $3 \mathrm{~s}, 90$ degree pulse length of $13.5 \mu \mathrm{s}$ at $21 \mathrm{~W}(-13.2 \mathrm{~dB})$, decoupling center of $1.39 \mathrm{ppm}$, PLW24 $=26.4 \mathrm{~dB}, \mathrm{P} 31=5$ $\mathrm{ms}, \mathrm{D} 31=0.5 \mathrm{~ms}, \operatorname{cpdprg} 2=$ hd.

3) HSQC (hsqcedetgp) : spectral width of $1050 \mathrm{~Hz}$ in a $1 \mathrm{~K} \times$ 128 (4 scans per increment, delay of $1.5 \mathrm{~s}$ ) matrix with $1 \mathrm{JCH}$ $=145 \mathrm{~Hz}$ and processed with pure cosine squared sine window functions and phase sensitivity using the echo/antiecho-TPPI mode \& CHn editing mode.

4) HMQC (hmqcgp) : spectral width of $1050 \mathrm{~Hz}$ in a $1 \mathrm{~K} \times 128$ (4 scans per increment, delay of $1.5 \mathrm{~s}$ ) matrix with $1 \mathrm{JCH}=$ $145 \mathrm{~Hz}$ and processed with pure cosine squared sine window functions and magnitude mode $(\mathrm{FnMODE}=\mathrm{QF})$.

By comparison, the pulse gradient duration used for 2D NMR is $1 \mathrm{~ms}$.

2. Optimal value of the decoupling center and decoupling width

It is important to find the optimal value of the decoupling center and decoupling width for efficient decoupling. The optimal decoupling center was identified to range from 1.38 to 1.40 ppm and zghd.3 had better for peak resolution than zghd.2 (Figures S1 and S2, respectively).

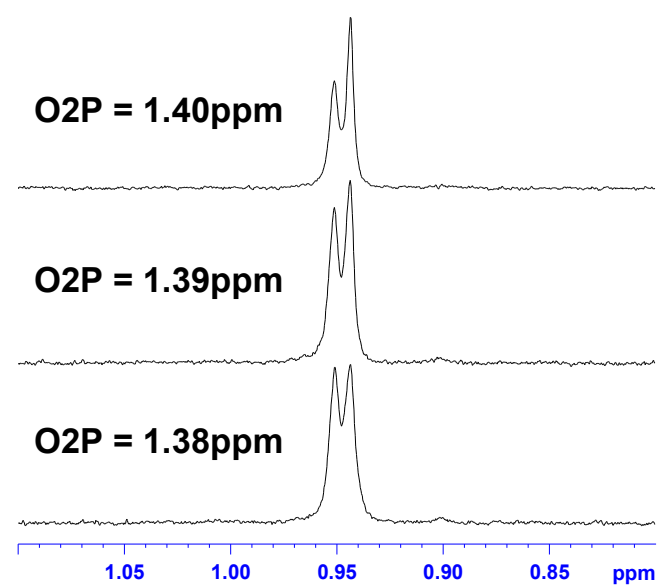

Figure S1. Identification of the optimal decoupling center (range from $1.38 \mathrm{ppm}$ to $1.40 \mathrm{ppm}$ )

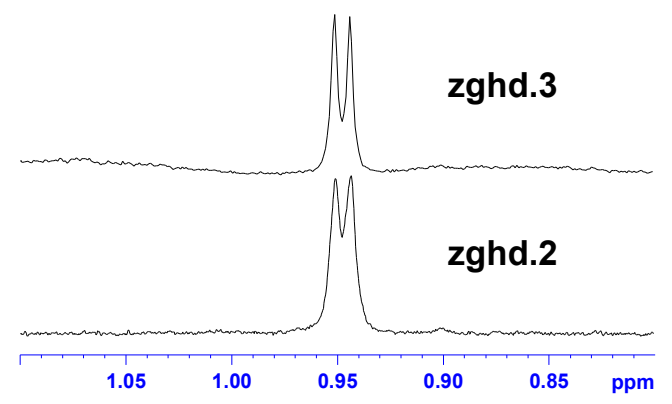

Figure S2. Identification of the optimal pulse program (zghd.3 has better resolution)

\section{Examples}

Example 1: Separation of methyl peaks between hexene and octene of EHOR

First, PE (EHOR, BASELL Inc.) containing 1-hexene and 1octene was melted with TCE- $\mathrm{d}_{2}$ at $100^{\circ} \mathrm{C}$. It is important to make homogeneous upon sampling; then, a $10 \mathrm{mg} / \mathrm{mL}$ sample was prepared and ${ }^{1} \mathrm{H}$ NMR spectra were acquired for 2 min at $100^{\circ} \mathrm{C}$.

Next, the optimal decoupling center is obtained by the homodecoupling pulse program (zghd.3).

First, the coupling pattern of the methyl peak was simplified to a singlet from a triplet by decoupling the methylene peak $\left(\mathrm{CH}_{2}\right.$, shown by pink arrow in Figure 3) coupling with the methyl group of 1-hexene and 1-octene at location number 2 .

By changing the optimal decoupling center from $1.30 \mathrm{ppm}$ to $1.42 \mathrm{ppm}$, the optimal center ranged from 1.38 to $1.40 \mathrm{ppm}$; by close observation, there was a marginal difference in the integral ratio between hexene and octene if the decoupling center was $1.38 \mathrm{ppm}$ or $1.39 \mathrm{ppm}$. However, the difference was approximately $10 \%$ at $1.40 \mathrm{ppm}$. Hence, considering its peak shape, the value of $1.39 \mathrm{ppm}$ was chosen as the decoupling center for EHOR.

Next, after setting the decoupling center as $1.39 \mathrm{ppm}$, under the conditions of PLW24 $=26.4 \mathrm{~dB}, \mathrm{P} 31=5 \mathrm{~ms}, \mathrm{D} 31=0.5$ $\mathrm{ms}$, cpdprg $2=\mathrm{hd}$, the integral value of 1 -hexene and 1-octene was calculated, as shown in Figure S3. 


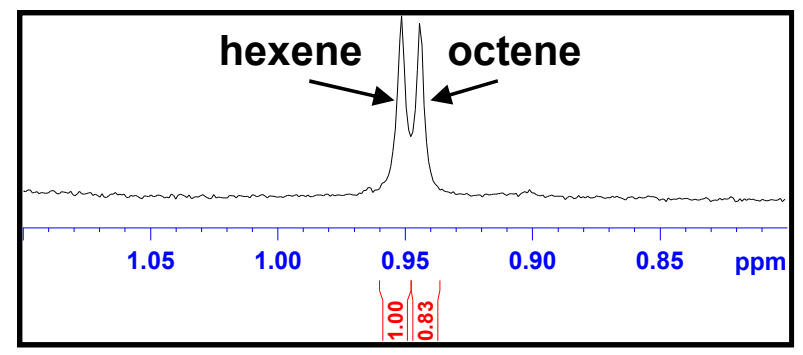

Figure S3. Homodecoupling ${ }^{1} \mathrm{H}$ NMR spectrum of EHOR (methyl region)

Integral ratio was hexene $:$ octene $=1.00: 0.83$, and it was calculated by the equation, $\mathrm{R}_{\mathrm{Hex}}=1.00 /(1.00+0.83)$, and $\mathrm{R}_{\mathrm{Oct}}$ $=0.83 /(1.00+0.83)$.

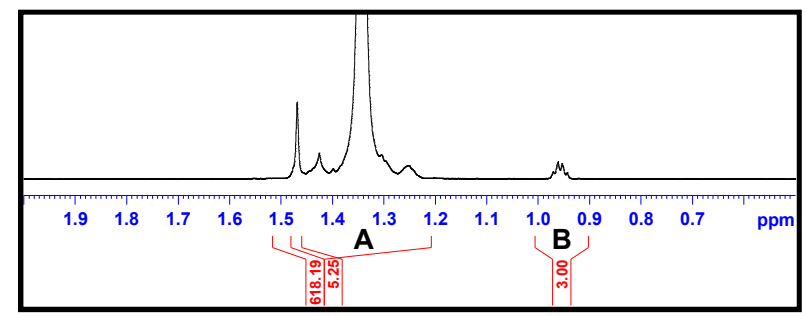

Figure S4. ${ }^{1} \mathrm{H}$ NMR spectrum of EHOR

Next, as indicated in Figure S4, after the measurement of the ${ }^{1} \mathrm{H}-\mathrm{NMR}$ peak, the molar ratio of the ethylene, 1-hexene, and 1-octene was calculated by utilizing the integral value of each domain.

Ethylene (molar ratio): 1-hexene (molar ratio): 1-octene (molar ratio)

$=\left[\mathrm{A}-\left(\left(\mathrm{B} \times \mathrm{R}_{\mathrm{Hex}}\right) / 3\right) \times 9-\left(\mathrm{B} \times \mathrm{R}_{\mathrm{Oct}}\right) \times 13\right] / 4:\left(\mathrm{B} \times \mathrm{R}_{\mathrm{Hex}}\right) / 3:$ $\left(\mathrm{B} \times \mathrm{R}_{\mathrm{Oct}}\right) / 3$

The number of branches in 1-hexene using previously obtained molar ratio is as follows.

The number of branches in 1-hexene

$=[$ molar ratio $(1$-hexene $) \times 1000] /[$ molar ratio $($ ethylene $) \times 2$

+ molar ratio $(1$-hexene $) \times 6+$ molar ratio $(1$-octene $) \times 8]$

The number of branches in 1-octene

[molar ratio $(1$-octene $) \times 1000] /$ [molar ratio (ethylene $) \times 2+$ molar ratio $(1$-hexene $) \times 6+$ molar ratio $(1$-octene $) \times 8]$

Example 2: Separation of methyl peaks between propylene and butene in EHBPR

First, polyethylene (EHBPR, DNP Inc.) containing propylene and butene was melted with TCE- $\mathrm{d}_{2}$ at $100^{\circ} \mathrm{C}$. It is important to make homogeneous upon sampling; after preparing a 10 $\mathrm{mg} / \mathrm{mL}$ sample, NMR spectra were recorded for $2 \mathrm{~min}$ at 100 ${ }^{\circ} \mathrm{C}$.
Next, the sample was analyzed with the following parameters using the 2D proton-carbon heterocorrelation pulse program contained in s/w Topspin v3.2 (Bruker BioSpin GmbH)

cnst2 $[\mathrm{J}(\mathrm{XH})=145 \mathrm{~Hz}], \mathrm{ns}=4, \mathrm{~d} 1=1.5 \mathrm{~s}, \mathrm{TD}=1 \mathrm{~K} \times 128$

If 2D HMQC experiment, gradient parameters are as follows:

Duration $(\mathrm{p} 16)=1 \mathrm{~ms}$, recovery delay $(\mathrm{d} 16)=200 \mathrm{us}$

Gradient programs: gpnam 1=gpnam2=gpnam3=SMSQ10.100

Gradient ratios: gpz1 $=50$, gpz $2=30$, and gpz $3=40.1$

The evolution period is optimized to $\mathrm{d} 2(1 / 2 \mathrm{~J}(\mathrm{CH}))=3.45 \mathrm{~ms}$ and the following values according to point 1 was modified: o1 (1H offset), o2 (13C offset), $2 \mathrm{sw}$ (1H spectral width) and 1 sw (13C spectral width). For instance: o1 $904 \mathrm{~Hz}$, o $24790 \mathrm{~Hz}$, 2 sw 1.5 ppm, 1 sw 45 ppm

$\mathrm{SI}=1 \mathrm{~K}$ and $\mathrm{SI} 1=1 \mathrm{~K}$ and pure cosine squared sine window functions (WDW2 $=$ WDW1 $=$ QSINE) are applied to both dimensions $(\mathrm{SSB} 2=\mathrm{SSB} 1=0)$ using $\mathrm{MC} 2=\mathrm{QF}$.

If 2D HSQC experiment, gradient parameters are as follows:

Duration $(\mathrm{p} 16)=1 \mathrm{~ms}$, recovery delay $(\mathrm{d} 16)=200 \mathrm{us}$

Gradient programs: gpnam1=gpnam2= SMSQ10.100

Gradient ratios: gpz1 $=80$ and gpz $2=20.1$

The evolution period is optimized to $\mathrm{d} 4(1 / 4 \mathrm{~J}(\mathrm{CH}))=1.72 \mathrm{~ms}$ and $\mathrm{o} 1$ (1H offset), o2 (13C offset), $2 \mathrm{sw}$ (1H spectral width) and $1 \mathrm{sw}$ (13C spectral width) same as HMQC experiment.

$\mathrm{SI} 2=1 \mathrm{~K}$ and $\mathrm{SI} 1=1 \mathrm{~K}$ and pure cosine squared sine window functions (WDW2 $=$ WDW1 $=$ QSINE) are applied to both dimensions (SSB2=SSB1=2) using $\mathrm{MC} 2=$ echo-antiecho.

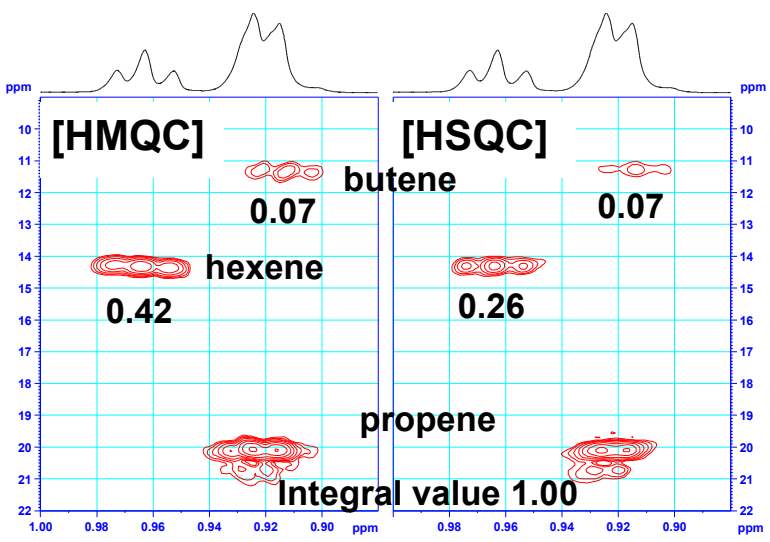

Figure S5. HMQC and HSQC spectra of EHBPR

Figure S5 shows the comparison of the results obtained by using the 2D pulse program hsqcedetgp (HSQC, phase sensitive \& $\mathrm{CHn}$ editing mode, FnMODE = echo-antiecho) and hmqcepqf (HMQC, magnitude mode, FnMODE $=\mathrm{QF}$ ). If the integral value of the methyl peaks from the respective monomers in ${ }^{13} \mathrm{C}$ - NMR was assumed to be true, the difference of 
the integral value between butene and propylene in HSQC and HMQC was within $10 \%$ after the comparison of the $2 \mathrm{D}$ peaks between HSQC and HMQC. In addition, the integral value of the measured spectrum by ${ }^{13} \mathrm{C}$ - NMR from polyethylene (EHBPR) including butene and propylene is shown in Figure S5; almost similar values were observed by comparison with the 2D peaks of HMQC or HSQC. Next, the integral ratio of butene and propylene was $1.00: 0.07$, as shown in Figure S5, and the integral ratio was calculated by $\mathrm{R}_{\mathrm{Pr}}=1.00 /(1.00+$ $0.07), \mathrm{R}_{\mathrm{Bu}}=0.07 /(1.00+0.07)$.

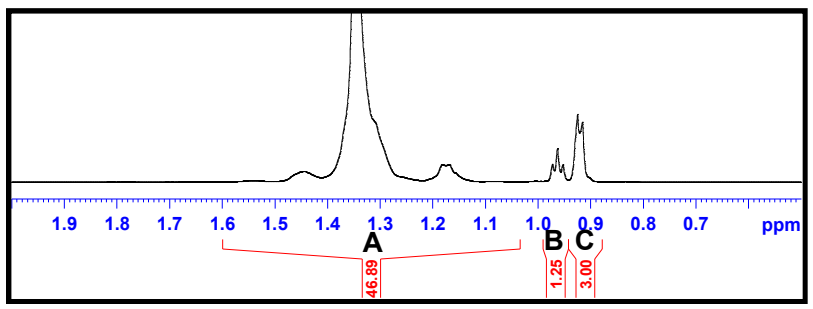

Figure S6. ${ }^{1}$ H NMR spectrum of EHBPR

Figure S6 shows the ${ }^{1} \mathrm{H}-\mathrm{NMR}$ peak of the sample, and the molar ratio of ethylene, 1-hexene, 1-butene, and 1-propylene using integral values can be calculated as follows.

Ethylene (molar ratio): 1-hexene (molar ratio): 1-butene (molar ratio): 1-propylene

$=\left[\mathrm{A}-(\mathrm{B} / 3) \times 9-\left(\left(\mathrm{C} \times \mathrm{R}_{\mathrm{Bu}}\right) / 3\right) \times 5-\left(\left(\mathrm{C} \times \mathrm{R}_{\mathrm{Pr}}\right) / 3\right) \times 3\right] / 4:$ $\mathrm{B} / 3:\left(\mathrm{C} \times \mathrm{R}_{\mathrm{Bu}}\right) / 3:\left(\mathrm{C} \times \mathrm{R}_{\mathrm{Pr}}\right) / 3$

The formula for calculating the number of branches of 1hexene using the achieved molar ratio is as follows.

The number of branches in 1-hexene

$=[$ molar ratio $(1$-hexene $) \times 1000] /[$ molar ratio $($ ethylene $) \times 2$

+ molar ratio $(1$-hexene $) \times 6+$ molar ratio $(1$-butene $) \times 4+$ molar ratio (1-propylene $) \times 3]$

The number of branches in 1-butene

$=[$ molar ratio $(1$-butene $) \times 1000] /[$ molar ratio $($ ethylene $) \times 2$ + molar ratio $(1$-hexene $) \times 6+$ molar ratio $(1$-butene $) \times 4+$ molar ratio (1-propylene) $\times 3$ ]

The number of branches in 1-propylene

$=[$ molar ratio $(1-$ propylene $) \times 1000] /$ molar ratio $($ ethylene $) \times$ $2+$ molar ratio $(1$-hexene $) \times 6+$ molar ratio $(1$-butene $) \times 4+$ molar ratio (1-propylene) $\times 3$ ]

Example 3: Separation of methyl peaks for hexene, heptene, and octene in LDPE

The methyl peak ratios for hexene, heptene, and octene in LDPE (LG Chem.), which contains all the branches from propylene, butene, hexene, heptene, and octene, were calculated in the same manner as demonstrated in example 1. Meanwhile, the methyl peaks ratios for propylene and butene were calculated in the same manner as in example 2. Thus, these two methods were combined to get the respective methyl peak ratio.
The methyl peaks of each hexene (C4), heptene (C5), and octene (C6) were separated using homo-decoupling, as shown in Figure $\mathrm{S} 7$.

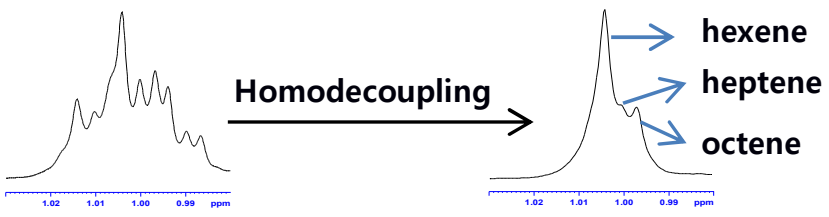

Figure S7. Homodecoupled ${ }^{1} \mathrm{H}$ NMR spectrum of LDPE (methyl region)

Even though the resolution was relatively low as compared to example 1, it was sufficient to obtain a ratio of the respective methyl peaks by integration.

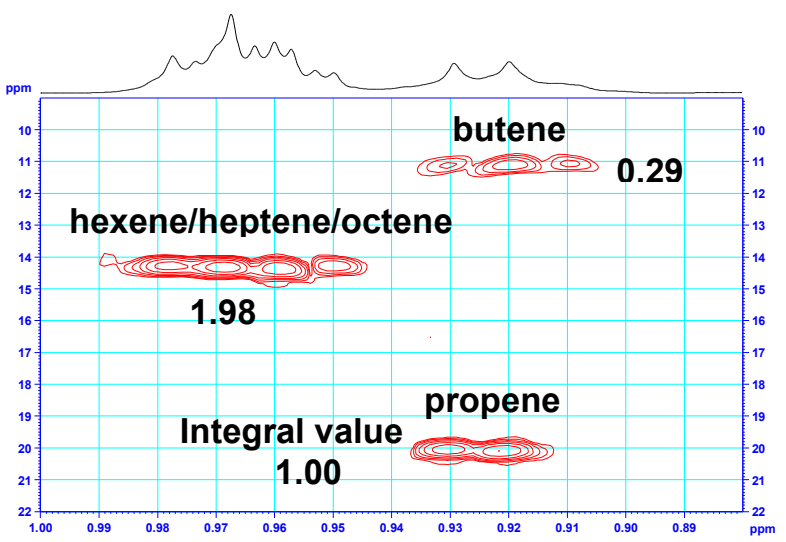

Figure S8. HMQC spectrum of LDPE

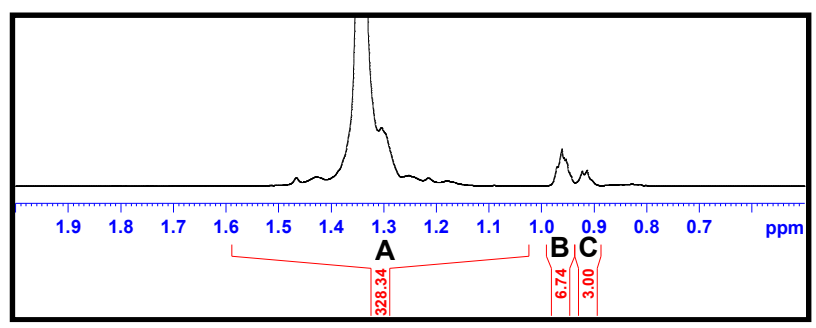

Figure S9. ${ }^{1} \mathrm{H}$ NMR spectrum of LDPE 


\section{Figures:}

Figure S1. Identification of the optimal decoupling center (range from $1.38 \mathrm{ppm}$ to $1.40 \mathrm{ppm}$ )

Figure S2. Identification of the optimal pulse program (zghd.3 has better resolution)

Figure S3. Homodecoupling ${ }^{1} \mathrm{H}$ NMR spectrum of EHOR (methyl region)
Figure S4. ${ }^{1} \mathrm{H}$ NMR spectrum of EHOR

Figure S5. HMQC and HSQC spectra of EHBPR

Figure S6. ${ }^{1} \mathrm{H}$ NMR spectrum of EHBPR

Figure S7. Homodecoupled ${ }^{1} \mathrm{H}$ NMR spectrum of LDPE (methyl region)

Figure S8. HMQC spectrum of LDPE

Figure S9. ${ }^{1} \mathrm{H}$ NMR spectrum of LDPE

- Additional text describing the parameters employed for NMR analysis, optimal value of the decoupling center and decoupling width, and three examples; three examples showing that separation of methyl peaks between hexene and octene of EHOR, methyl peaks between propylene and butene in EHBPR and methyl peaks for hexene, heptene, and octene in LDPE. 